\title{
COMMENTS ON OCEANOGRAPHIC INSTRUMENT DEVELOPMENT
}

\author{
By Carl Wunsch
}

\section{Oeanographic}

instrument

development is slow

and expensive-and

increasingly painful.
$\mathrm{O}$

CEANOGRAPHY is in large part an observational science. Physical oceanography in particular clearly shares with the rest of fluid dynamics a deep symbiosis of theory with observation. The governing equations of motion, while known, contain so many diverse balances and resulting phenomena that observation is required to guide theory. Thus, oceanography is replete with phenomena that could have been predicted but which were discussed dynamically only after they were observed (one thinks of microstructure, the equatorial undercurrents and the Gulf Stream recirculation). Or, even had they been predicted, it is quite likely that no theory' would have been taken seriously in the absence of clear observational evidence (I an trying to forestall letters pointing out that an obscure 19 th century paper predicted microstructure).

Oceanography is an excellent example of a science driven by technology. Although it is often argued that science drives technology, equally cogent arguments can be made in the other directionthat more often than not, new technology drives new science. The classic example is Galileo and the telescope; he neither invented that instrument nor wals the first to use it. But the way he applied the technology led to a scientific revolution.

Arguably, the most important advance in oceanography after the second World War was the invention of the transistor and its integrated-circuit descendants. Modern electronics made possible most of the instruments (current meters, floats and computers to analyze the results) that we now have. which have led to great strides in understanding of the ocean. The excitement and health of the science are dependent upon continued rapid development and evolution of our instruments. Intellectual leadership in oceanography traditionally has lain with those countries and institutions at the technical cutting edge.

U.S. oceanography has been notable for the ingenuity and yeastiness of its instrument and engineering development. I was motivated to write this note by an increasing sense that in this country we may be

Carl Wunsch. Department of Earth. Amospheric and Planetary Sciences, Massachusets Institute of Technology, Cambridge. MA 12139 losing the ability to maintain the degree of activity and progress we have had in the past. There is no immediate cause for alarm, but in the long-term there could be serious problems.

\section{Is Anything Wrong?}

Although I have not done any kind of systematic survey, I have the strong sense that the symbiotic relationship between ocean scientists and engineers is breaking down. It is fairly easy to point out the problems: it is much more difficult to find remedies. But identifying the problems is at least a first step.

Oceanographic instrument development is slow and expensive-and increasingly painful. It is slow for two connected reasons and expensive for two connected reasons. The fundamental slowness lies in the nature of the occan. Increasingly. many of the problems oceanographers are grappling with demand ever longer measurement times; ocean physics are "slow," and the ocean noisy. It, for example, one wishes to study the monsoon response of the western Indian Ocean, the phenomenon is only quasi-periodic on the annual time scale; elementary statistics suggest many years, if not decades, of observation are needed. Thus, one faces the need for developing instruments which can survive for years. Demonstrating that they do survive for years takes years.

But consider the even earlier stages - merely showing that one "s new instrument "works" at all. In a typical cycle. a scientist if technically competent, or in collaboration with an engineer, will have an idea for a new instrument. A proposal is written and sent off. If the scientist is expeditious. and fortunate with deadlines and reviewers, roughly a year from conception he might have some money to build a prototype. Perhaps building and testing the prototype takes a year (this estimate is extremely optimistic; if bench testing of new sensors is required, at least one or two more years are required at this stage). One then needs to show that the instrument works. Perhaps one finds shiptime to get the instrument into the water six months later, and if it is an unattended instrument. perhaps it is left in the water to show that it operates for a month. Often there is some failure; one goes back. makes changes, re-tests and finds shiptime for another trial. Being optimistic, the science/engineering team assumes that cure of the first failure means 
that all is now "right," that the instrument works. Thus, the next trial is a year in duration. often under the guise of doing some science with it. For a whole year, there is often no information at all (if the instrument. as is typical of moored ones. only records data internally). One then fetches the instrument, and often there has been another failure. At this point there is a real crisis. The first block of funds (usually for no more than 3 years) has run out: the scientist has to go back to the well for more money. with a failed instrument, and empty-handed except for his own faith in its ultimate worth, to face skeptical, sometimes scathing peer-reviewers and program managers. With luck, he gets to try again in another cycle.

I suspect that typically any really novel instrument takes a minimum of 1 wo such cycles to arrive at the point where the originating scientist has something that at least some people agree "works." At that point, he gets to use the instrument to do some science. In practice, the time scales to go from conception to truly operational use are much longer. Stommel first described a SOFAR (Sound Fixing and Ranging) float in 1954. but it was only about 1974 in the hands of Rossby and Webb that it became a serious scientific tool. Hamon and Brown began building STDs (instruments to measure salinity. temperature and depth) in the early 1950s, but they were hardly commonplace much before 1970. There are many other such examples (c.g.. the time to develop a reliable subsurface mooring and a useful current meter to go on it ).

Unfortunately. two cycles or six years is the time to tenure review in major research universities. It is also longer than the job duration of most funding agency program managers. The original manager, enthusiastic about the potential new instrument, has long since been succeeded by another who can t understand why all that money was being poured down what appears to be a bottomless rathole.

The technology is expensive (pressure vessels. mooring cables, mass stores, acoustic gear, ships and crews. etc.). Failures are thus not only conspicuous. they also lead to understandable recriminations from funding agencies and colleagues about wasted resources. But if failures are not tolerated, no one will ever develop a really new instrument. Industry estimates that to support one ordinary engineer takes about $\$ 200.000$ per year (including salary, overhead and perhaps one technician). Add to that all the ancillary expenses of an oceanographic research group, and multiply it by ten years, and large sums of money are being conspicuously consumed.

A theoretician, or numerical modeler, can quietly toss his mistakes into the trash can with no one the wiser (how much computer output represents "failed" experiments that no one but the practitioner is ever aware of?).

In response to this situation, institutions, individuals and funding agencies have begun to draw back from instrument development. Parts of the U.S. Navy (I am not referring to ONR) quite clearly decided that modeling is cheap and observations are expensive and drew the conclusion that the right strategy was to put money into modeling in the innocent belief that with a good enough model one would not need very many observations to make operational forecasts. Of course, the situation is precisely the opposite. The more sophisticated the model, the greater the demand for observations to initialize it and to provide boundary conditions. The Navy accepts that it takes over twenty years to take a weapons system from conception to fleet deployment: it has lost patience with oceanographic systems whose development time is half that-in the incessant demand for instant conversion of science into fleet-deployable capabilities.

\section{What Can Be Done?}

For technical developments, the three-year proposal cycle is too short: it commits the principal investigator to a relentless, ncarly unforgiving schedule built on a "success-assumed" logic. The comparatively scanty sums with which the engineering groups must operate ultimately probably cost more because of the higher incidence of failure and the extra time incurred. Herein lies a tricky political problem: serious novel instrument development grants and contracts should probably run nominally for about seven years with a "light" mid-term review. But the L.S. government makes budgets on an annual basis; that the National Science Foundation and the Office of Naval Research have been able to juggle their own budgets to permit three and occasionally five year commitments is a small miracle in itself. Is there nonetheless some way to permit longer funding cycles? The whole nature of oceanography with its multi-year time scales requires a much longer programmatic commitment than even three years.

Much of oceanographic science is developing in the direction of large-scale. long-duration programs: the Ocean Drilling Program (ODP), Global Ocean Flux. World Ocean Circulation Experiment. Although many deplore these developments as incompatible with the traditional methods of oceanography, the emergence of such programs reflects the increasing global sweep of the science and the necessity of tackling processes on ever longer time scales. With the exception of ODP. none of these programs is yet actually fully launched. or even funded. Nonetheless. these programs all have planning horizons which run out a decade and longer; all envision the need for technologies which do not yet exist in practice. One possibility for both providing the tools these programs need and the funding stability required for instrument development is to build into them a "technology tax." Each should carry programmatically some significant fraction of their overall budgets directed at a decade-long investment in the instruments needed only at theirend-an investment directed primarily at the successor gencration of oceanographic programs (ODP already does this to at degree, so that the precedents exist).

| PI.FAsE TLRA TOPAGil 64 |

\section{For technical}

developments, the

three-year proposal

cycle is too short. 


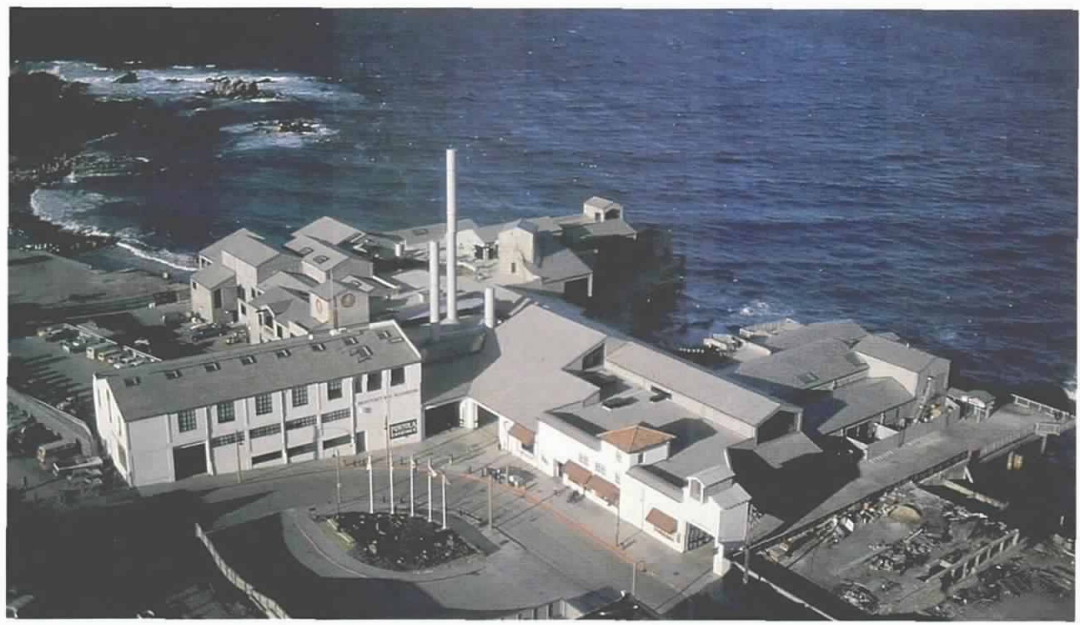

(c) 1989, MONTEREY BAY AQUARIUM

\section{COMMENTS ON OCEANOGRAPHIC INSTRUMENT DEVELOPMENT}

Continued From Page 27

The major oceanographic institutions also need to find ways to support innovative instrument development engineers, in close collaboration with scientists, but with their own reward system and encouragement to innovate on their own. There are a number of clever engineers to be found in the oceanographic community, but they are not very conspicuous; they are often confused with "applied oceanographers," and they are rarely recognized even by their own institutions (at least one of our large oceanographic institutions insists that all its staff must be labelled "scientists" even if they are really engineers - a rather brutal statement of values). Engineering culture is not oriented toward publication the way the science culture is; measures of achievement appropriate for a physical oceanographer-meant to be doing and publishing science-are often inappropriate for an engineer, whose product is a new instrument that someone else will use to do science. Indeed, one good measure of a successful engineer is the number of people, outside the original group, who have successfully used one of his new instruments. Some modified reward structure, established to sustain innovative oceanographic instrument development, would surely go a long way toward ameliorating this situation. It is true that oceanographic-instrument engineering, if done independently of working scientists, can go off into sterile directions (the same is true of theory done in isolation from observations). But there are good, creative instrument engineers with the common sense to stay in close touch with the scientists who would need to work with the data. At the present time, the insti- tutional/funding system almost demands that such people can at best be junior partners of the scientists.

To encourage true engineering innovation, the system has to be willing to invest in a few talented individuals and bet on their ideas for about a decade at a time. It would be expensive-but all field oceanography is expensive-and probably cheaper in the long run than the present piecemeal, short-term system.

There are probably other, better remedies than these. Perhaps The Oceanography Society could take a lead in trying to define ways to sustain the technical developments which seem so central to the health of our science.

Acknowledgement: D.J. Baker provided some especially useful comments.

\section{DUAL-CAREER COUPLES AND SCIENCE: OPPORTUNITIES, CHALLENGES AND STRATEGIES Continued From Page 31}

Rossi, A.S., 1965: Women in science: Why so few? Science 148, 1196-1202.

Rossiter, M.W., 1982: Women Scientists in America: Struggles and Strategies to 1940. Johns Hopkins University Press, Baltimore, $439 \mathrm{pp}$.

Schwartz, F.N., 1989: Management women and the new facts of life. Harvard Business Revien 67(1), 65-76.
U.S. Office of Personnel Management, 1988: Helping Federal Employees Balance Work and Family Life. Report to the President, OPM Doc. 149 79-9, October 1988.

University of Wisconsin, 1988: Achieving Faculty Diversity: A Sourcebook of Ideas and Success Stories. University of Wisconsin System, Madison, $52 \mathrm{pp}$.
Webster, B.D., 1989: Opening doors for women in academia. Bioscience 39, 96-98.

Weishaar, M., K. Chiaravalli and F. Jones, 1984: Dual-career couples in highereducation.Journal of the National Association for Woman Deans, Administrators, and Counselors (NAWDAC) 47, 120-124.

Widnall, S.E., 1988: AAAS Presidential Lecture: Voices from the pipeline. Science 241, 17401745. $\square$ 